\title{
Sismo de Capellades 01 diciembre 2016, Cartago, Costa Rica
}

\section{Capellades earthquake 2016-12-01, Cartago, Costa Rica}

\author{
Ronnie Quintero-Quintero ${ }^{1}$ \\ Hernán Porras-Hernández ${ }^{2}$ \\ Universidad Nacional, Costa Rica
}

\begin{abstract}
Resumen
En este trabajo se hace una descripción de la fuente sísmica del sismo de Capellades, provincia de Cartago, Costa Rica, del 1 de diciembre de 2016, 00:25:20 hora UTC. Se estudian los parámetros de la fuente, centroide, momento, magnitud, mecanismo focal; así como hypocentro de las réplicas y sus magnitudes. También se hace una descripción de la sismicidad en la zona epicentral antes del sismo de Capellades. Los datos usados en el análisis fueron registrados por la red sísmica del Observatorio Vulcanológico y Sismológico de Costa Rica de la Universidad Nacional (OVSICORIUNA) y el Laboratorio de Ingeniería de la Universidad de Costa Rica (LIS-UCR). El sismo principal se localizó con una profundidad hipocentral de $3.0 \mathrm{~km}, 3.7 \mathrm{~km}$ al NNO de Capellades, con una magnitud $(\mathrm{Mw})$ de 5.2. Éste sismo se ubica entre los volcanes Turrialba e Irazú; Cordillera Volcánica Central de Costa Rica. La solución del tensor de momento indica una falla transcurrente dextral, casi vertical (buzamiento $82^{\circ}$ ), rumbo $155^{\circ}$ y deslizamiento $174^{\circ}$; con un centroide a 6 $\mathrm{km}$ de profundidad. El eje de presión está indicado por un strike y dip de $21^{\circ} / 1^{\circ}$; es decir, con una dirección SSO-NNE y el de tensión de $111^{\circ} / 10^{\circ}$; indicando una tensión en la dirección SEE-NOO. En general, el mecanismo focal obtenido usando inversión de ondas o el impulso de los primeros arribos indican una falla transcurrente, con ejes de presión y tensión horizontales en concordancia con los esfuerzos de otros eventos sísmicos intraplaca moderados y ligeros que han ocurrido en el
\end{abstract}

1 Académico, Observatorio Vulcanológico y Sismológico de Costa Rica, Universidad Nacional, Heredia, Costa Rica. Correo electrónico: rquinter@una.cr

2 Académico, Observatorio Vulcanológico y Sismológico de Costa Rica, Universidad Nacional. Heredia, Costa Rica. Correo electrónico: porrashernan@gmail.com

Este artículo corresponde a la ponencia presentada en el I Congreso Centroamericano de Ciencias de la Tierra y el Mar, realizado en San José, Costa Rica, del 13 al 16 de noviembre de 2017. 
arco volcánico central de Costa Rica. En 5 días de actividad sísmica, en la zona comprendida entre 9.9 y 10.12 latitud norte, 83.9 y 83.72 longitud oeste, se localizaron 1923 sismos; todas las réplicas con magnitudes menores a 4.0 grados en la escala Richter. La relocalización de las réplicas muestra una tendencia epicentral con dirección NO-SE, con buzamiento al SO y concentrada en un área de $32 \mathrm{~km}^{2}$. El rumbo de $155^{\circ}$ coincide con la tendencia mostrada por los epicentros de las réplicas, lo que indica el azimut de traza de la falla. La zona del Irazú-Turrialba, donde ocurrió el sismo del 1 de diciembre 2016, mostró microsismicidad meses y años antes del sismo principal, pero no se tenía conocimiento de sismos similares a éste, en el área de ruptura.

Palabras clave: Costa Rica, Sismo Capellades, tensor de momento; réplicas

\begin{abstract}
In this work a seismic source description of the Capellades, Cartago province, Costa Rica earthquake, 2016-12-01 00:25:20 UTC time is made. We study the fault's parameters, centroid, moment, magnitude, focal mechanism and aftershocks hypocenter with respective magnitudes. We also made a seismicity description in the epicentral zone before the Capellades earthquake occurred. The seismic network of the Observatorio Vulcanológico y Sismológico de Costa Rica de la Universidad Nacional (OVSICORI-UNA) and the Laboratorio de Ingeniería de la Universidad de Costa Rica (LIS-UCR) recorded the data used in the analysis. The main earthquake was located at $3.0 \mathrm{~km}$ depth, $3.7 \mathrm{~km}$ to the NNO of Capellades, with a magnitude $(\mathrm{Mw})$ of 5.2. This earthquake was located between the Turrialba and Irazu volcanoes, Central Costa Rica Volcanic Arc. The moment tensor solution indicates a dextral strike-slip, almost vertical (dip $82^{\circ}$ ), strike $155^{\circ}$ and slip $174^{\circ}$, with a centroid depth at $6 \mathrm{~km}$. The pressure axis was calculated with a strike and dip of $21^{\circ} 1^{\circ}$, with an SSO-NNE direction and the tension axis with strike and dip of $111 \% 10^{\circ}$, indicating a tension in the SEE-NOO direction. In general, the focal mechanism obtained using waveform inversion or P-wave first motion indicate a strike-slip fault, with horizontal pressure and tension axes in accordance with the stress of other moderate and light intraplate seismic events that have occurred in the central volcanic arc of Costa Rica. In 5 days of aftershock activity, in the area between 9.9 and 10.12 north latitude, 83.9 and 83.72 west longitude, 1923 earthquakes were located, all with magnitudes less than 4.0 degrees on the Richter scale. The aftershocks relocation shows an epicentral trend with NW-SE direction, dipping to the SW and rupture area of $32 \mathrm{~km}^{2}$. The strike $155^{\circ}$ coincides with the tendency shown by the aftershocks epicenters, which indicates the fault trace. The Irazu-Turrialba zone where occurred the December 1, 2016 earthquake, showed microseismicity months and years before the main event; but there was no knowledge of earthquakes like this one, in the area.
\end{abstract}

Keywords: Costa Rica, Capellades earthquake, moment tensor, aftershocks

\title{
Tectónica y sismicidad previa al sismo de Capellades
}

La actividad sísmica interplaca e intraplaca en Costa Rica son consecuencia de la interacción de las placas de Coco, Nazca, Caribe y Microplaca de Panamá (Meschede and Barkhausen, 2000; Marshall et al., 2000). La distribución de la sismicidad a lo largo del contacto entre las placas Coco y Caribe sugiere que el límite entre ambos se caracteriza por la alternancia de segmentos de subducción planos y normales (De Mets, 2001; Protti et 
al., 1995). La placa del Coco se subduce normal a la trinchera con una tasa de convergencia de $91 \mathrm{~mm} / \mathrm{a}$ a $82 \mathrm{~mm} / \mathrm{a}$ y dirección de convergencia de $025^{\circ}-030^{\circ}$ (Bird, 2003; De Mets et al., 2010). El ángulo de subducción de la placa del Coco produce variaciones en los esfuerzos en la placa superior, que se caracterizan por la generación de sismos de magnitudes locales intermedias $(5,0$ y 6,5$)$ con foco superficial, como los que se producen en la zona central del país. Zona que se conoce como el cinturón deformado del centro de Costa Rica (CCRDB); que sería parte de la frontera occidental del Bloque de Panamá y se caracteriza por una serie de fallamientos activos (Marshall et al., 2000).

Desde que entró en operación la Red Sísmica del OVSICORI-UNA en el año 1984, ha registrado una gran cantidad de sismos superficiales en la zona central del país, la mayoría sólo perceptible por instrumentos sísmicos (ver Figura 1). Aunque han sido pocos los sismos que han ocurrido con magnitud menor a $6.5 \mathrm{Mw}$ en la parte central del país, si han sido de intensidad fuerte a violenta. Antes del sismo de Capellades del $01 / 12 / 2016$, dos sismos fuertes y superficiales fueron ubicados en la zona central del país, el terremoto de Puriscal del 22/12/1990, Mw=6.0 y el de Cinchona, $\mathrm{Mw}=6.1$, ubicado en el macizo del volcán Poás el 08/01/2009 (Segura et al., 2014). El sismo de Capellades del 01/12/2016 fue sentido en el Valle Central, causó pequeños deslizamientos y daños en 60 casas en la zona epicentral, y puede catalogarse como un sismo de intensidad fuerte (Campos \& Quintero, 2017). 
Figura 1. Sismicidad superficial registrada por la red sísmica del OVSICORI-UNA en el Centro de Costa Rica; de 1984 a diciembre 2015.

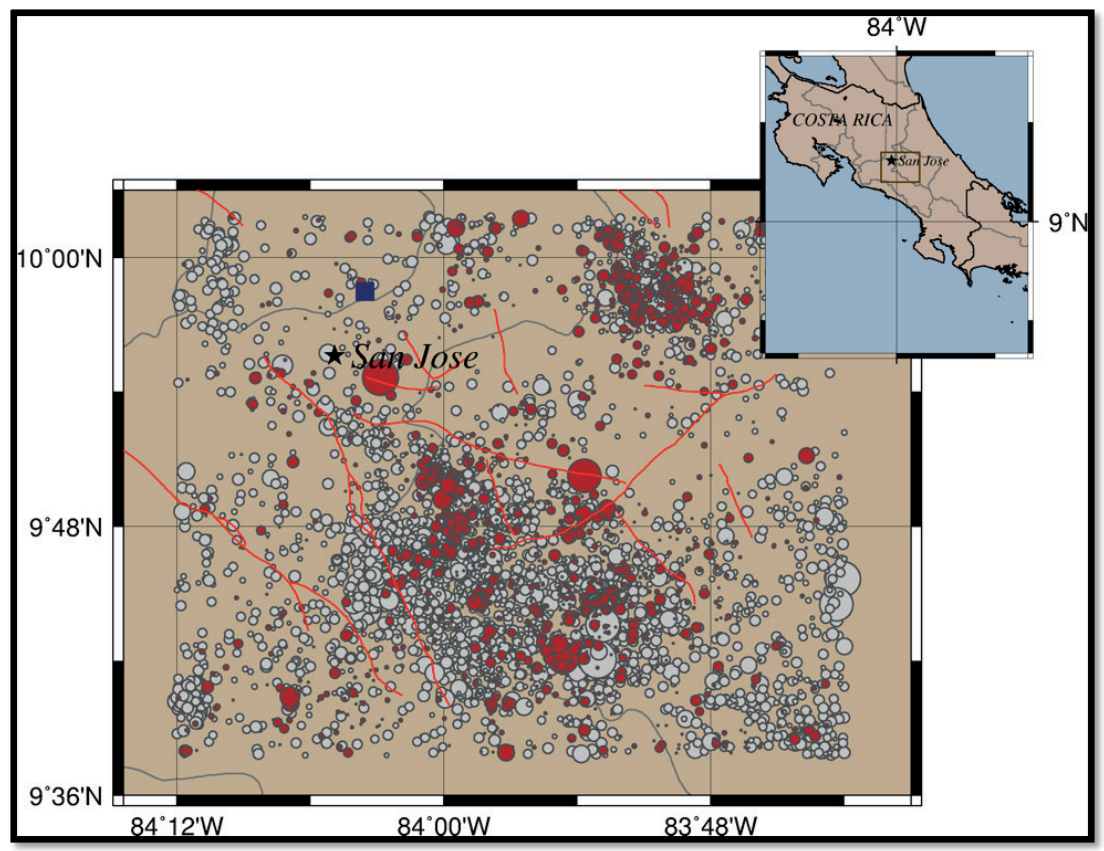

Los sismos en color gris son eventos antes del 2010 y en rojo sismos registrados después de ese año. Con líneas de color rojo se indican fallas inferidas o mapeadas (Montero, 1999; Rojas et al., 1998, Fernández y Rojas, 2001; Denyer, et al., 2003). Con una estrella de color negro se indica la ciudad de San José.

El sismo de Capellades, ocurre entre los macizos del volcán Irazú y Turrialba; zona en que la red sísmica del OVSICORI-UNA localizó un total de 3111 sismos superficiales antes del 1 de diciembre del 2016, con 1017, sismos entre el 2010 y el 30/11/2016. La mayoría de estos sismos se ubicaron en la cercanía del cráter del Irazú.

Muchos de los sismos localizados en la Cordillera Volcánica Central del país se han presentado en forma de enjambre sísmico, como fue la sismicidad registrada en el macizo del volcán Irazú en el 2010; de éstos, el sismo de mayor magnitud fue de $3.2 \mathrm{Ml}$ y se registró el 22 de julio a las 05:51 a.m., hora local, y se ubicó 7 km al SE del cráter del volcán Irazú. Durante el 2010 y 2011, la red del OVSICORI-UNA localizó 141 sismos en el complejo Irazú-Turrialba, todos con magnitud menor a M1 3.7. 
La actividad sísmica y superficial en la zona central del país se mantuvo durante el 2012; en enero se localizaron varios sismos en las cercanías de San Juan de Chicua, Cartago, comunidad ubicada al suroeste del cráter del Volcán Irazú, la sismicidad fue superficial con profundidades menores a los $5 \mathrm{~km}$ y magnitudes que no superaron los 3.6 grados Ml. En total se localizaron 138 sismos en los macizos del Irazú y Turrialba durante ese año.

Para el 2013, la actividad sísmica en el complejo volcánico Irazú Turrialba fue de poca frecuencia y el sismo de mayor magnitud localizado en esa zona tuvo una magnitud M1 3.4 y ocurrió el 18/12/2013 22:35:47. Se registraron un total de 23 sismos en ese complejo volcánico. Para el 2014 se incrementó la cantidad de sismos localizados en la zona comparada al del 2013, se contabilizaron 107 microsismos en el complejo IrazúTurrialba, todos con magnitud menor a Ml 2.9.

Durante el 2015 se localizaron 1136 sismos en el complejo volcánico Irazú-Turrialba, con la mayoría de ellos ubicados al SO del cráter del volcán Irazú. Desde el inicio de enero se localizaron sismos de baja magnitud y superficiales que fueron sentidos por la población local. Para el 25 de mayo de 2015, se presenta una actividad sísmica superficial asociada a fallamiento local, ubicada $4.5 \mathrm{~km}$ suroeste del cráter del Irazú. Al ser las 02:19:38 del 26 de mayo, se reporta un sismo sentido y superficial en Potrero Cerrado de Oreamuno, M1 2.6. Después de este sismo se registraron varios sismos de magnitud baja en la zona y el 26/05/2015 02:50:28 se localiza un sismo que fue sentido en la zona epicentral, Cartago Centro y en la capital San José, con magnitud Ml 4.0; pero que no causó daños.

Desde enero al 30 de noviembre de 2016, se localizaron 546 sismos en el complejo Irazú-Turrialba; cantidad baja comparada con la localizada en el 2015 en la zona. El estudio de esta sismicidad no muestra un patrón o premonitor que nos indicara que se produciría un sismo fuerte en la zona.

\section{Sismo principal y réplicas}

La red sísmica del OVISCORI-UNA empezó el monitoreo de la actividad sísmica y volcánica de Costa Rica en abril de 1984, y para finales del 2016 la mayoría de las estaciones sísmicas con la que contaba eran digitales. El paquete ANTELOPE se usa a partir del 2010 para almacenar, localizar y distribuir datos sísmicos (Segura et al., 2014) y fue el que se utilizó en la localización del sismo principal y réplicas. Específicamente, se usó el 
programa Genloc (Fan et al., 2006) implementado dentro de ANTELOPE y el modelo de velocidad de Quintero \& Kissling (2001) para la localización de los sismos e inversión de las formas de onda. En la localización de esta actividad sísmica se usaron estaciones de la red símica permanentes del OVSICORI-UNA, de una red temporal instalada por los autores desde el año 2010 en la zona y datos que comparte en tiempo real el LIS-UCR (http:// www.lis.ucr.ac.cr; Moya, 2006) con el OVSICORI-UNA. En total 67 estaciones fueron usadas para el análisis de la sismicidad asociada al sismo de Capellades (ver Figura 2). Los autores leyeron y localizaron todos los sismos de los cinco primeros días del mes de diciembre de 2016 ubicados en la zona y mostrados en este estudio.

Figura 2. Estaciones usadas en el estudio del sismo de Capellades del 01/12/2016 y réplicas (triángulos de color gris). Una gran cantidad de estaciones se encuentran en la zona central del país y cercanas a los volcanes Irazú y Turrialba.

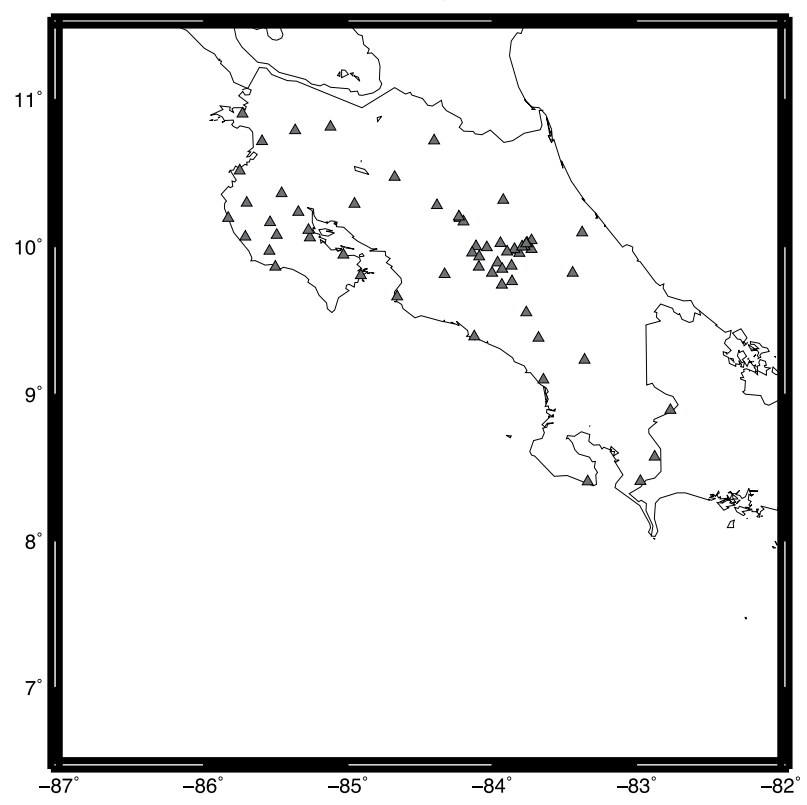

Como se menciona arriba, el número de sismos localizados hasta el 30 de noviembre de 2016 eran pocos comparados con los del 2015; esa tendencia se rompe a partir del 1 de diciembre; cuando se presenta un 
sismo fuerte (00:25:20 hora UTC), localizado $3.7 \mathrm{~km}$ al Noroeste de Capellades de Alvarado, Cartago, Cordillera Volcánica Central de Costa Rica.

El sismo principal se localiza con 62 lecturas de la onda P; de éstas, 8 estaciones se ubicaron a una distancia menor a $10 \mathrm{~km}$. Las lecturas de la onda $\mathrm{S}$ se tomaron de los acelerómetros OCM, RIMA, CDM del OVSICORI-UNA y HCPD, SLMA, SASR del LIS, estaciones ubicadas a una distancia entre 20 y $45 \mathrm{~km}$ del epicentro. Para la localización de las réplicas se usaron las estaciones más cercanas a la zona epicentral, la cual se compone de 19 estaciones sísmicas; las cuales estaban instaladas en el momento que se da la sismicidad en la zona (ver Figura 2) y se usan para el monitoreo de los procesos volcánicos del Irazú y Turrialba, así como estaciones temporales usadas para el monitoreo sísmico de la sismicidad en la zona de Cartago. En total se relocalizaron 1923 sismos en los primeros cinco días del mes de diciembre del 2016 y los hipocentros se concentran en un área de $8 \mathrm{~km}$ de largo y $4 \mathrm{~km}$ de profundidad, lo cual nos da una idea del área $\left(32 \mathrm{~km}^{2}\right)$ de ruptura del sismo principal (Figura 3). El uso de redes sísmicas locales permitió localizar las réplicas con muy buena precisión, donde el 98\% de los hypocentros tienen elipses de error con semiejes mayor y menor que no sobrepasan los $2 \mathrm{~km}$ de error. El sismo principal es uno de los sismos con elipse de error de menor tamaño. Se puede notar de la Figura3, que las elipses de error tienen una tendencia del semieje mayor con dirección NO, indicando que la red de estaciones usadas en la localización tiene una apertura menor en esa dirección; en nuestro caso, gran cantidad de estaciones se ubican en el macizo del volcán Turrialba e Irazú, pero no al norte del Irazú; por lo que el acimut del semieje mayor de la elipse de error tiene dirección $\mathrm{NO}$ y el semieje menor dirección NE. Las réplicas muestran un plano casi vertical y buzando hacia el SOO (Figura3C), lo cual nos da una idea del ángulo de inclinación de la falla y dirección. La Figura3B nos muestra pocas réplicas en los tres primeros km del hipocentro hacia el NO y hacia abajo, que señala el área donde se concentró la mayor parte del deslizamiento del evento principal. La localización de las réplicas nos indica que la ruptura es hacia el NO del hipocentro del sismo principal y el tiempo de ruptura es corto y dura menos de 3 segundos (GCMT, 2018). Las elipses de error de las localizaciones y mostradas en la Figura3 no distorsiona el patrón de la sismicidad y la distribución hacia el $\mathrm{NO}$ e inclinación hacia el SOO de los hypocentros. 
Figura 3. Mapa epicentral de la sismicidad asociada al sismo de Capellades del 1 de diciembre de 2016 (Figura 3A). Los epicentros de las réplicas se muestran por medio de círculos rellenos de color rojo; el tamaño es acorde a su magnitud. El epicentro y centroide del sismo principal se muestran por medio de estrellas de color negro. Para el centroide se muestra su mecanismo focal. Los volcanes Irazú y Turrialba se indican por triángulos de color gris. Los pueblos más cercanos a la zona epicentral se indican por cuadrados de color celeste. Las Figuras 3B y $3 \mathrm{C}$ muestran los hypocentros proyectados a lo largo de la línea continua mostrada en la figura 3A y perpendicular a ésta; respectivamente. En las figuras $3 \mathrm{~B}$ y $3 \mathrm{C}$ los sismos se indican por puntos de color negro y la localización del sismo principal y su centroide por medio de estrellas de color rojo. El centroide es el que se ubica a $6 \mathrm{~km}$ de profundidad. En la figura se muestran las elipses de error del $98 \%$ de los sismos.

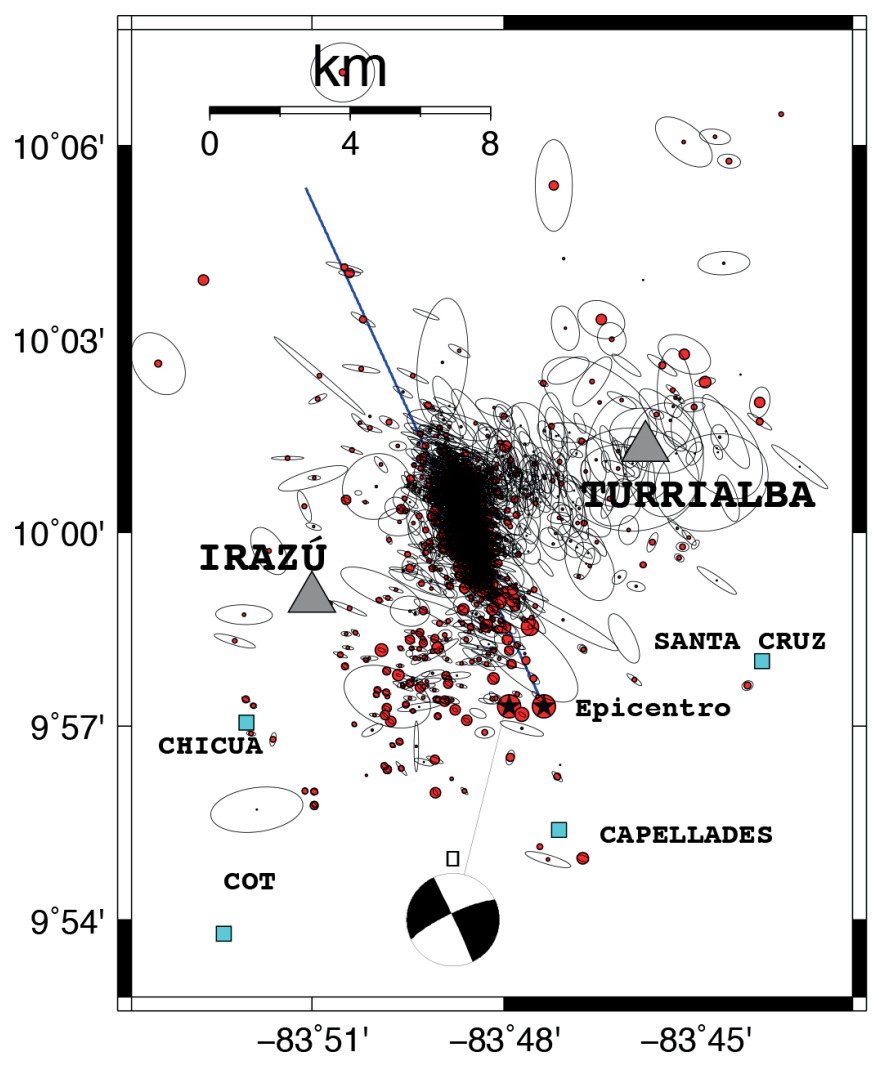


Ronnie Quintero-Quintero - Hernán Porras-Hernández

Sismo de Capellades 01 diciembre 2016, Cartago, Costa Rica

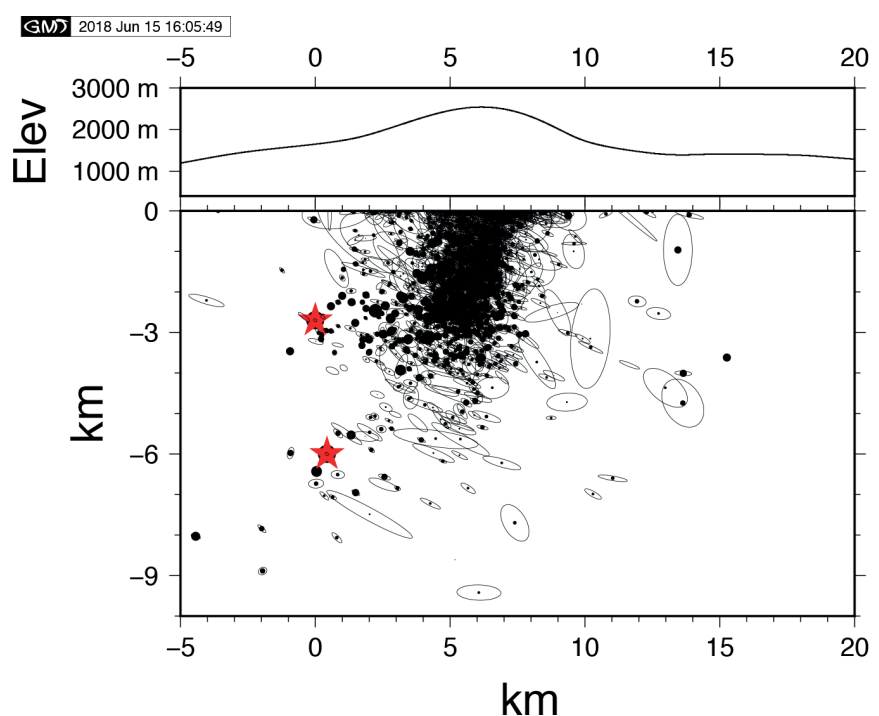

B

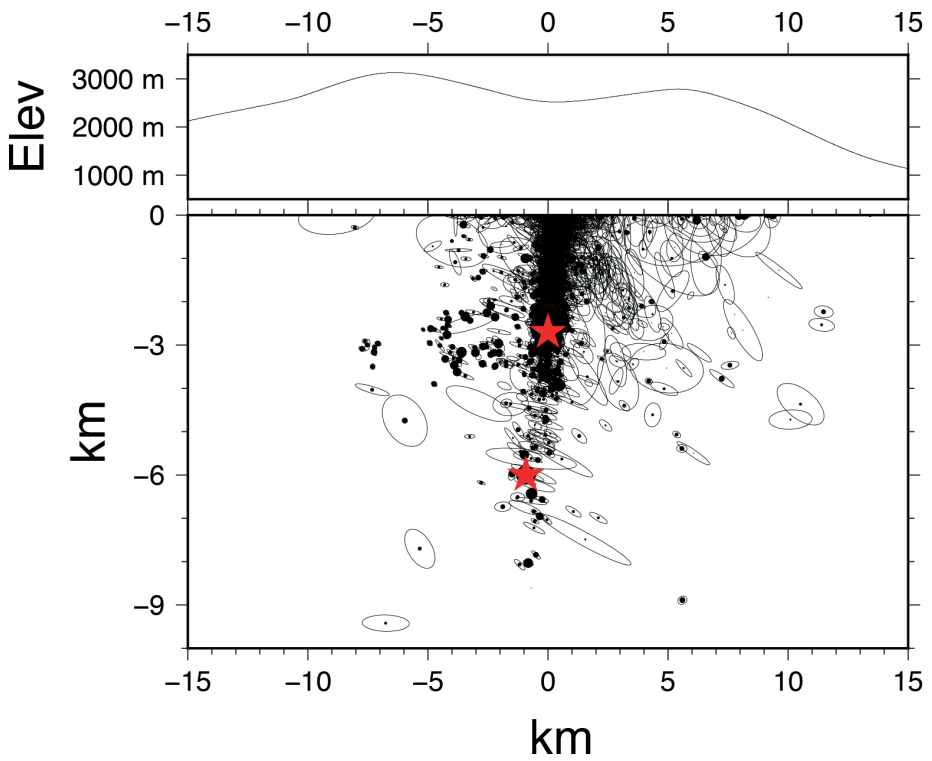

Este sismo tiene las características de un sismo principal con sus réplicas, las cuales decaen en número rápidamente y se mantienen altas sólo durante los primeros días después del evento principal. Para el quinto día, la cantidad de sismos vuelve a caer a un nivel mostrado antes del sismo principal (Figura 4A). Las réplicas, mostradas en la Figura 3A, tuvieron 
magnitud baja, oscilando entre 0 y 4 grados en la escala Richter, la mayoría con magnitudes entre 0.75 y 2 grados $\mathrm{Ml}$ y pocos con magnitud mayor a 3 grados Ml (Figura 4B).

Figura 4. Número de sismos localizados por día en la zona IrazúTurrialba (A); comenzando con los sismos localizados el 26 de noviembre de 2016. Se observa que para el 2016-12-05 el nivel de sismicidad se compara con la mostrada en los últimos días de noviembre

de ese año. En la figura 4B, se muestra el histograma de la magnitud Richter (Ml) de la sismicidad de Capellades; para los cinco primeros días de diciembre de 2016.

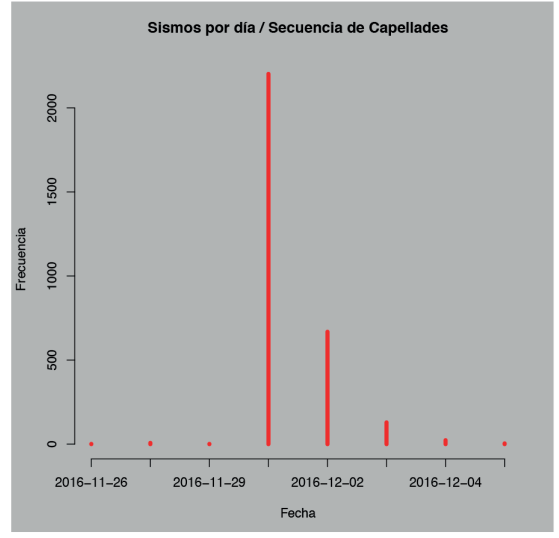

A

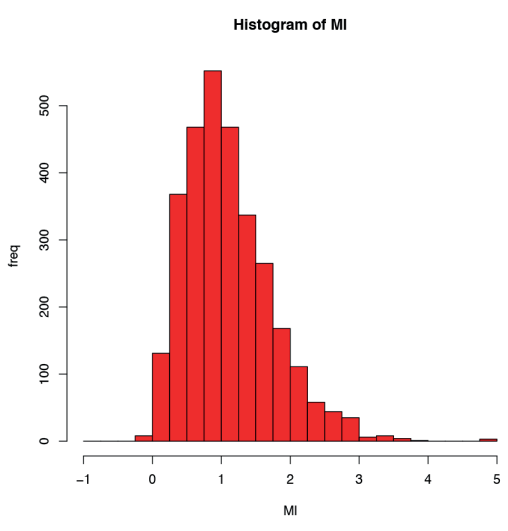

B

El mecanismo focal del evento principal obtenido usando las polaridades del primer impulso de la onda $\mathrm{P}$, nos indica claramente que se trata de un sismo producto de una falla transcurrente (Figura 5) y que está en concordancia con el mecanismo focal obtenido usando inversión de ondas sísmicas (Figura 6). La traza de falla indicada por una solución del mecanismo focal obtenido con las polaridades indica un rumbo NO-SE $\left(151^{\circ}\right)$, con buzamiento alto $\left(66^{\circ}\right)$ y hacia el SO. Dado que este plano nodal está en concordancia con el patrón de localización de las réplicas, se asume como el plano de la falla. 
Figura 5. Mecanismo focal derivado de la polaridad de los primeros impulsos del sismo del 01-12-2016 a las 00:25:20 horas UTC. El eje de Presión está indicado por la letra $\mathrm{P}$ y el de tensión por la letra T. Mecanismo focal derivado usando el programa FOCMEC (Snoke et al., 1984). Con strike, dip y rake de $151^{\circ} / 66^{\circ} / 160^{\circ}$ con eje de presión $\mathrm{P} 19^{\circ} / 5^{\circ}$ ( en dirección SSO-NNE) y eje de tensión T $111^{\circ} / 30^{\circ}$ (en dirección SEE-NOO). Las estaciones con respectiva polaridad se indican a los lados de la esfera focal; con triángulos se indica dilatación y con círculos compresión.

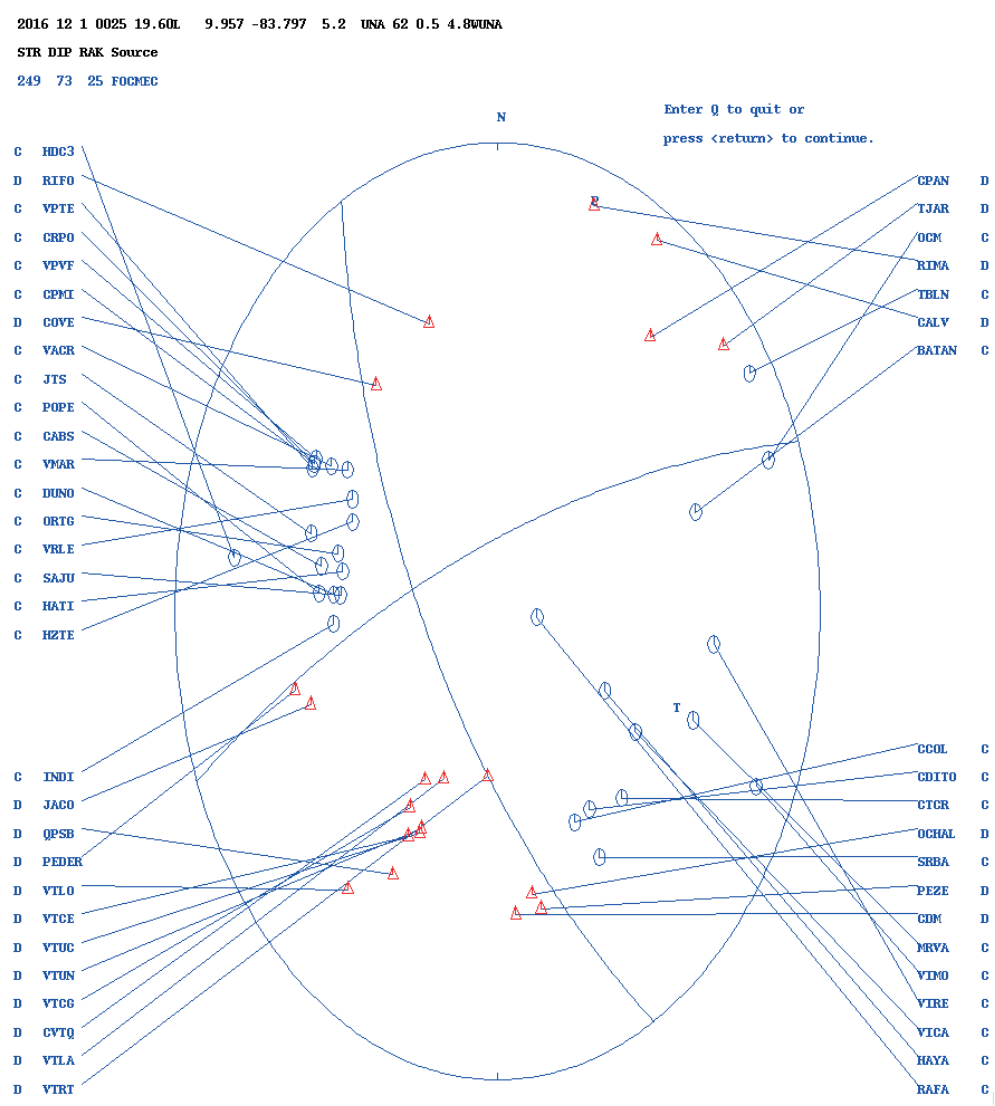

Después de calculado el mecanismo focal usando polaridades, usamos datos de formas de onda de las estaciones de banda ancha del OVSICORI-UNA, para calcular el Tensor de Momento (CMT) puntual. Usamos 
diferentes combinaciones de estaciones de banda ancha que se encuentran entre 43 y $188 \mathrm{~km}$ del epicentro; con un rango de frecuencia entre $0.02 \mathrm{a}$ $0.06 \mathrm{~Hz}$. Aquellas estaciones que presentan perturbación de baja frecuencia en el instrumento (Zahradník and Plešinger, 2005) por el paso de las ondas del sismo o que no tienen una buena relación señal-ruido, no se usan en la inversión y para la estación JTS se hace la respectiva rotación para tener componentes EW, NS y Z. La inversión fue realizada usando el paquete ISOLA (Sokos and Zahradník, 2008, 2013) y la búsqueda del centroide se hizo vertical y horizontalmente (en 45 puntos de un plano de tamaño $3 \mathrm{kmx} 2 \mathrm{~km}$ ). Formalmente, se encuentra el centroide $3 \mathrm{~km}$ hacia abajo y $1 \mathrm{~km}$ al oeste del hypocentro; pero la correlación entre los datos reales y sintéticos en cualquier punto del plano horizontal de tamaño $3 \mathrm{~km} \times 2 \mathrm{~km}$ es alta y casi de 0.9 . El centroide se ubica hacia abajo y al oeste del hipocentro, 0.12 segundos después, relativo al tiempo de origen y a 6 $\mathrm{km}$ de profundidad (ver Figura 3); con un plano nodal (rumbo $155^{\circ}$, buzamiento $82^{\circ}$ y ángulo de deslizamiento de $174^{\circ}$ ) que concuerda con los hypocentros de las réplicas, porcentaje de par acoplado de $97 \%$, Mw 5.2 y una alta correlación entre las formas de onda reales y sintéticas, con una reducción de la varianza global $\mathrm{VR}=0.8$ (Figura 6 y 7). La magnitud de momento aumenta si se toman las estaciones más distantes del epicentro, ya que se puede bajar el contenido de frecuencia a una banda de 0.02 a 0.5 Hz. Se realizaron pruebas de estabilidad de la solución, realizando inversión de las formas de onda para las siete estaciones (CDM, RIFO, BATAN, JACO, COVE, JTS, CDITO), donde en cada inversión no se tomaba en cuenta una estación; los resultados muestran un intervalo para el ángulo de buzamiento de $73^{\circ}-85^{\circ}$, rumbo $151^{\circ}-156^{\circ}, 243^{\circ}-246^{\circ}$ y ángulo de deslizamiento $164^{\circ}-173^{\circ}$ y $5^{\circ}-19^{\circ}$, lo que indica un alto grado de estabilidad de la inversión (ver Figura 9). 
Ronnie Quintero-Quintero - Hernán Porras-Hernández

Sismo de Capellades 01 diciembre 2016, Cartago, Costa Rica

Figura 6. Tensor de momento del sismo del 01 diciembre de 2016, calculado usando ISOLA (Sokos and Zahradník, 2008, 2013). Eje P está definido por strike/dip de $21^{\circ} / 1^{\circ}$ y el eje T por $111^{\circ} / 10^{\circ}$. En el mapa se muestra la esfera focal y estaciones usadas para su cálculo.

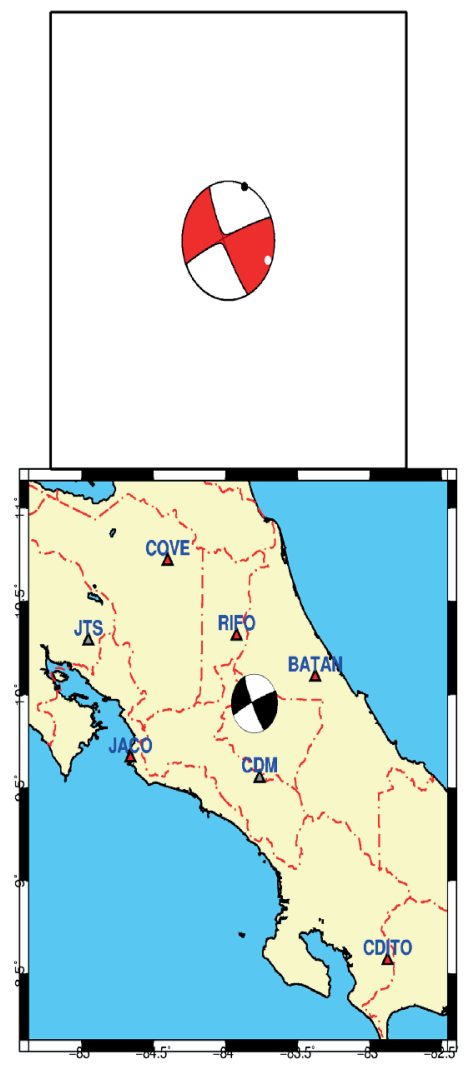

MOMENT TENSOR SOLUTION

HYPOCENTER LOCATION (UNA)

Origin time $2016120100: 25: 20.00$

Lat 9.9551 Lon -83.7895 Depth 3

\section{CENTROID}

Trial source number : 13 (Multiple Source line or plane inversion)

Centroid Lat (N) 9.9551 Lon (E) -83.7986

Centroid Depth $(\mathrm{km}): 6$

Centroid time : +0.12 (sec) relative to origin time

Moment (Nm) : 7.771e+16

Mw : 5.2

VOL\% :0

DC : 97.1

CLVD $: 2.9$ SNR CN FMVAR STVAR.

$\begin{array}{llllll}\text { Var.red.: (for stations used in inversion):0.8 } & \text { NaN } & 2.7 & 8 \pm 4 & 0.69\end{array}$

Var,red. (for all stations) $\quad: 0.76$

Strike Dip Rake | Frequency band used in inversion (Hz)

$\begin{array}{lllll}155 & 82 & 174 & \text { | } & 0.02-0.06\end{array}$

Strike Dip Rake I Stations-Components Used-Distance

$\begin{array}{llllll}246 & 84 & 8 & \text { I } & \text { NS EW Z } & \mathrm{D}(\mathrm{km})\end{array}$

|RIFO +++43

P-axis Azimuth Plunge ICDM - -44

$\begin{array}{lll}21 & 1 & \text { BATAN } t++48\end{array}$

T-axis Azimuth Plunge |JACO +++101

$\begin{array}{llll}111 & 10 & \mathrm{COVVE} & +++108\end{array}$

-

Mrr Mtt Mpp ICDITO + + 183

$0.338 \quad-5.889 \quad 5.551$

Mrt Mrp Mtp

$-0.644-1.150 \quad 5.085$

Exponent (Nm): 16 
Figura 7. Correlación de las formas de onda de desplazamiento reales (negro) y sintéticas (rojas) para el sismo del 01/12/2016 00:25:20 en un ancho de banda de 0.02-0.06 Hz. La nomenclatura de las estaciones se indica en la izquierda. El gráfico para cada componente de la estación sísmica indica tiempo (segundos) y su desplazamiento (metros). En cada cuadro se indica la reducción de la varianza.

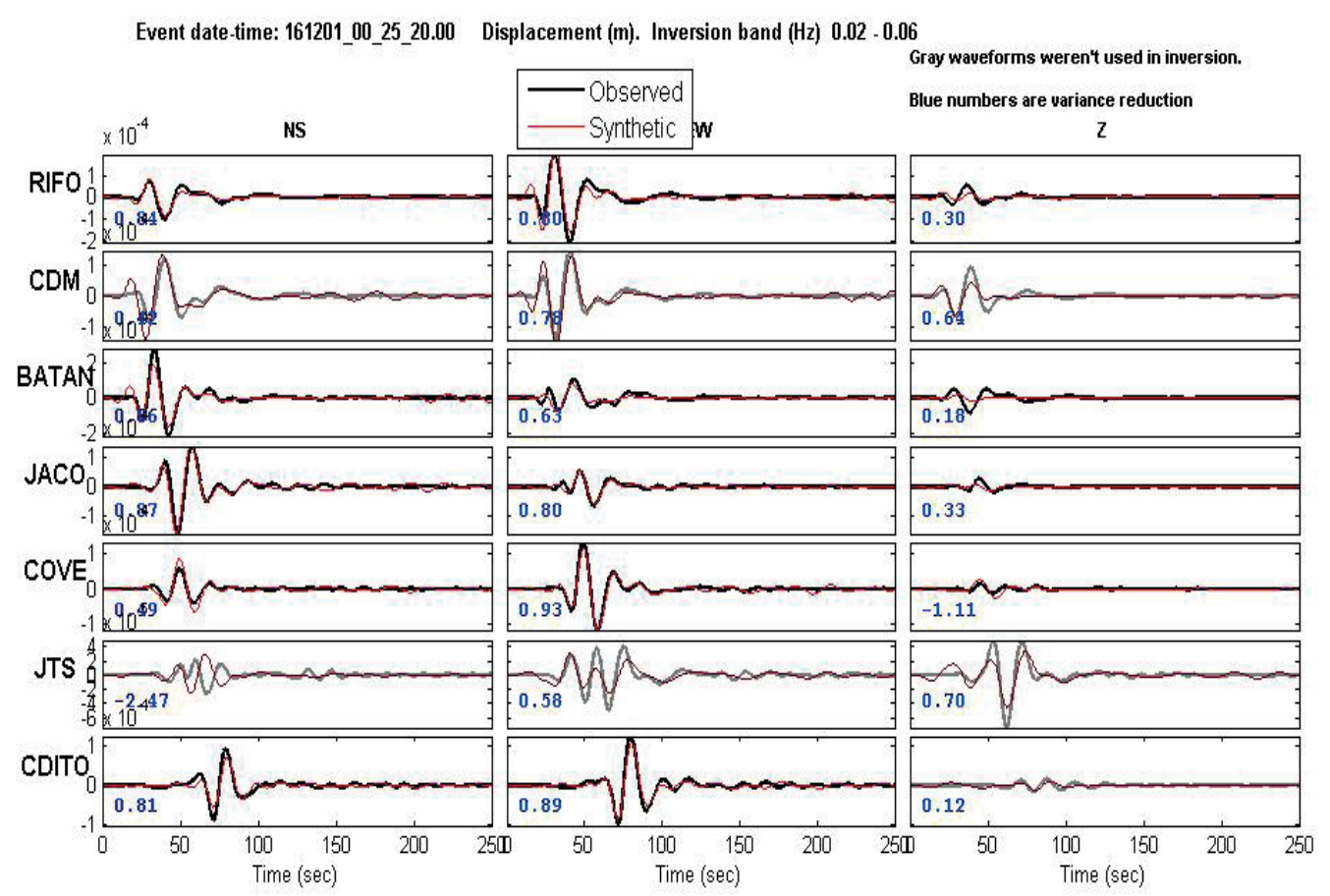

Aunque la sismicidad se encuentra cercana al complejo volcánico Irazú - Turrialba; durante las primeras 24 horas después del sismo principal, la actividad sísmica no disparó cambios en la actividad volcánica que venía presentando el Volcán Turrialba los días previos; la cual consistía principalmente de desgasificación, con pluma de color blanco indicando salida de vapor de agua y tremor de baja amplitud (http://www.ovsicori. una.ac.cr; https://www.facebook.com/OVSICORI/). 


\section{Discusión y conclusiones}

- $\quad$ Las réplicas del sismo de Capellades del 01/12//2016 00:25:25 horas UTC se ubican en los primeros $9 \mathrm{~km}$ de profundidad desde el nivel del mar, con la mayoría concentradas en los primeros $4 \mathrm{~km}$ y el estudio del tensor de momento (Figura 6) nos muestra que el sismo tiene mayor contenido de un doble-par de fuerzas (97\%) que de no doble-par de fuerzas ( $3 \%$ ), lo cual nos hace concluir que el sismo es tectónico y no volcánico y el sismo principal no mostró correlación directa con el proceso eruptivo del volcán Turrialba, volcán que se encontraba en proceso eruptivo durante ese año.

- $\quad$ Casi todas las réplicas se ubican hacia el NO del epicentro con un largo de $8 \mathrm{~km}$, lo cual indica un rompimiento hacia una sola dirección y presentan una disminución grande de magnitud con respecto al evento principal, concentrándose con magnitudes menores a tres grados en la escala Richter. La característica principal del sismo es que se muestra como un típico sismo principal con réplicas; las cuales vuelven a una actividad de fondo que existía antes del evento principal, a partir del quinto día.

- La localización de las réplicas indica que el buzamiento del plano de falla es casi vertical con ligera inclinación hacia el suroeste y por lo tanto con rumbo de la traza de falla NO-SE, transcurrente dextral. También se puede concluir que el tamaño de la falla es pequeño, con un área de ruptura de $32(8 \times 4) \mathrm{km}^{2}$. La mayoría de las réplicas ocurren del hypocentro hacia la superficie.

- Los pocos daños que causó el sismo principal están relacionados directamente con el tamaño del sismo Mw 5.2 y su corta duración de 2 seg (GCMT, 2018)

- $\quad$ La sismicidad localizada antes del sismo de Capellades del 2016 se presentaba con magnitudes bajas y algunas veces en forma de enjambres sísmicos y la mayoría localizada al SO del cráter principal del volcán Irazú (Figura 8). No se encontraron indicadores premonitores del sismo principal.

- La concentración de las réplicas a lo largo de una línea de falla; así como la sismicidad localizada en el complejo volcánico Irazú-Turrialba para el período 2010-2016 (Figura 8) nos hacen escoger el plano nodal (strike $155^{\circ}$, dip $82^{\circ}$ y rake $174^{\circ}$ ) como el plano de falla. 
Aunque anteriormente, otros fallamientos habían sido mapeados en la zona (Lupi at al., 2014), la falla delimitada por la concentración de réplicas del sismo de Capellades de unos $8 \mathrm{~km}$ de largo no había sido mencionada antes del sismo del 01/12/2016. Por lo tanto, el conocimiento de la falla de unos $8 \mathrm{~km}$ de largo, con $4 \mathrm{~km}$ de profundidad y con orientación hacia el SE es importante para futuros análisis de amenaza sísmica en la región.

- La cantidad de datos usados en la localización de los sismos nos dan soluciones hypocentrales que tienen los semiejes de la elipse de error de localización muy pequeños, y estas elipses no afectan la distribución espacial de los sismos; lo cual nos da confianza en seleccionar un plano nodal con dirección NO-SE y buzando al SW y ángulo casi vertical.

- La semejanza entre las soluciones del mecanismo focal obtenido usando polaridades e inversión de las formas de onda, nos da un alto grado de confiabilidad del tensor de momento calculado, lo cual indica que el centroide se ubica hacia abajo $(3 \mathrm{~km})$ y hacia el oeste $(1 \mathrm{~km})$ del hypocentro. En general, la alta correlación que existe en la inversión, para cualquier punto entre el hypocentro y el centroide nos indica que el plano que forman está contenido por los hypocentros de las réplicas; también, la frecuencia máxima de $0.06 \mathrm{~Hz}$ usada en la inversión de ondas tiene una longitud de onda mayor a $16 \mathrm{~km}$, por lo que no debe de existir mayor diferencia entre la posición del hypocentro y del centroide. De igual forma, el grado de estabilidad del centroide se comprueba realizando repeticiones de la inversión por el mecanismo focal donde cada vez se elimina una estación en la inversión y donde el ángulo de rotación entre cada solución obtenida de K-angle (Sokos \& Zahradník, 2013) es pequeño (Figura 9). 
Figura 8. Mapa con los epicentros de la sismicidad superficial registrada por la red sísmica del OVSICORI-UNA en la zona cercana a los volcanes Irazú - Turrialba desde el 2010 al 2016. Los epicentros se indican por círculos rellenos de color gris o rojo. Los epicentros de color rojo son los asociados a la sismicidad de Capellades. Los volcanes se muestran por triángulos de color rojo. El tamaño del círculo es acorde a la magnitud. El mecanismo focal se indica por una esfera focal. Los pueblos cercanos de Chicúa, Cot, Santa Cruz y Capellades se indican por cuadrados de color celeste.

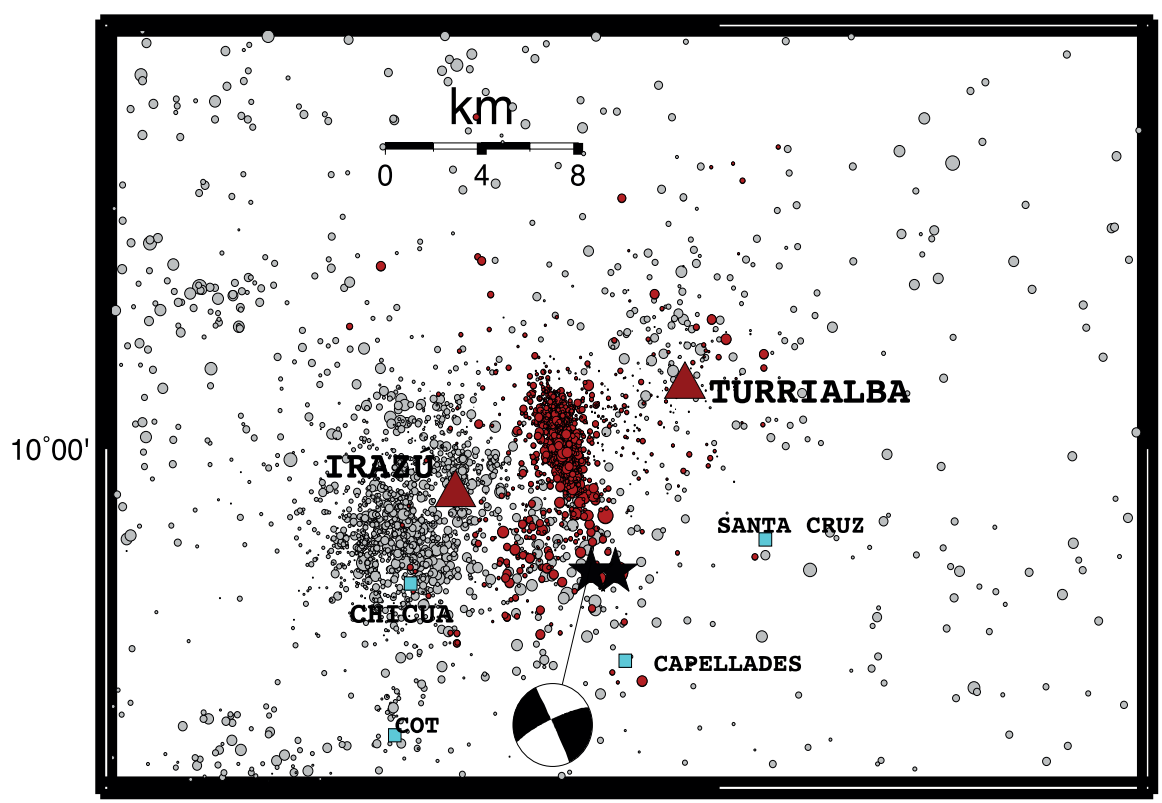

$-83^{\circ} 45^{\prime}$ 
Figura 9. Evaluación de la incertidumbre de los tres ángulos de la fuente strike, dip, rake, de la posición de la fuente en 45 puntos de un área de $3 \mathrm{~km} \times 2 \mathrm{~km}$, ubicada $3 \mathrm{~km}$ por debajo de la profundidad hypocentral, tiempo del centroide relativo al tiempo de origen hypocentral, porcentaje de DC, planos nodales y el ángulo-K.
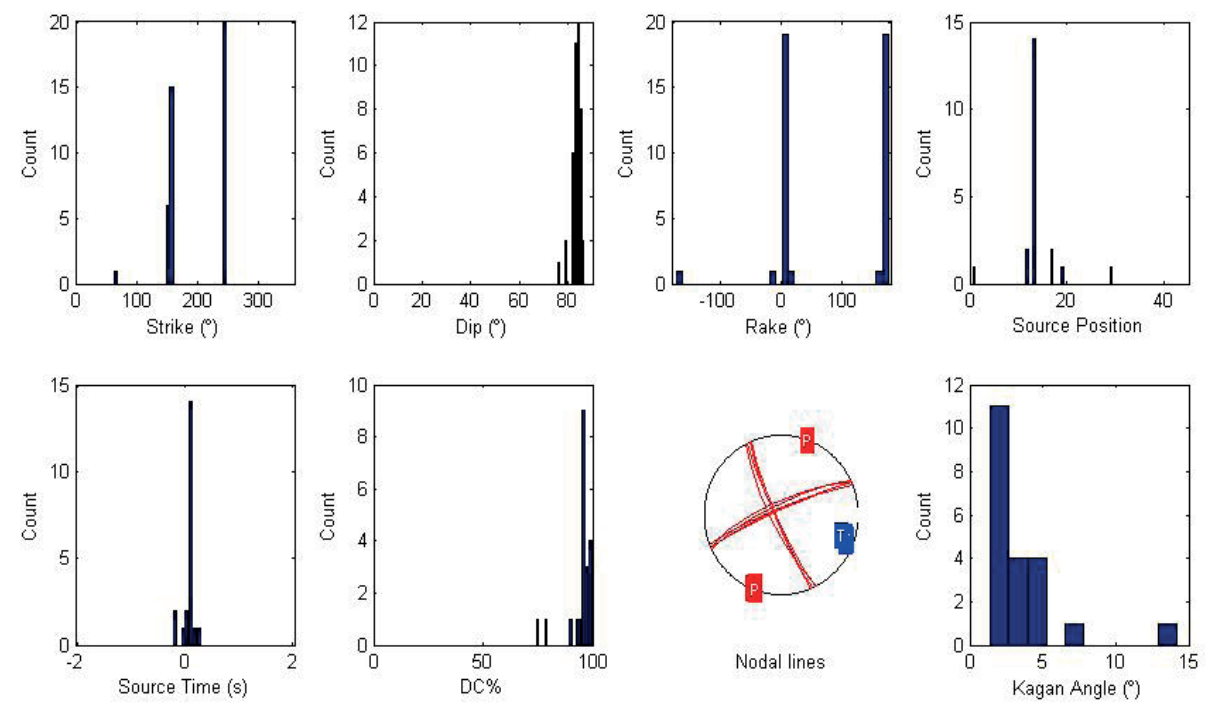

\section{Agradecimientos}

Se agradece al Gobierno de Costa Rica por el financiamiento para instalación y el mantenimiento de las estaciones sísmicas del OVSICORI-UNA. Se agradece al LIC-UCR por compartir datos de aceleración en tiempo real con el OVSICORI-UNA. Se agradece al equipo de profesionales del OVSICORI-UNA por mantener en operación la red sísmica y por el trabajo en los catálogos sísmicos del país, desde 1984. La base de datos originales usads para el artículo se encuentran en la base de datos del OVSICORI-UNA. Las figuras se generaron usando el programa computacional GMT (Wessel and Smith, 1995), SEISAN (Havskov and Ottemöller, 1999), R (R Core Team, 2013) e ISOLA (Sokos and Zahradník, 2008, 2013). Se agradece a Lucas Barros de la Universidad de Brasilia, Brasil y Jiri Zahradník de Charles University, Prague, República Checa por sus comentarios y sugerencias que ayudaron a mejorar el artículo. 
Ronnie Quintero-Quintero - Hernán Porras-Hernández

Sismo de Capellades 01 diciembre 2016, Cartago, Costa Rica

\section{Referencias}

Campos, D., Quintero, R. (2017). Mapa de Intensidades e implicaciones socioambientales del sismo de Capellades. Nov. 30, 2016; Costa Rica. I Congreso Centroaméricano de Ciencias de la Tierra y el Mar, 13 al 16 de Noviembre, 2017. San José, Costa Rica.

Bird, P. (2003). An updated digital model of plate boundaries. Geochem. Geophys. Geosyst. 4(3), DOI:10.1029/2001GC000252.

Denyer, P., W. Montero, and G. E. Alvarado (2003). Atlas Tectónico de Costa Rica, Editorial Universidad de Costa Rica, serie Reportes Técnicos, Costa Rica.

De Mets, C. (2001). A New Estimate For Present-Day Cocos-Caribbean Plate Motion: Implications For Slip Along The Central American Volcanic Arc.- Geophys. Res. Letters, 28: 4043-4046.

De Mets, C., Gordon, R.G. \& Argus, D.F. (2010). Geologically Current Plate Motions. Geophys. J. Int. 181: 1-80.

Fan, C., G. L. Pavlis, and K. Tuncay (2006). GCLGRID: A three-dimensional geographical curvilinear grid library for computational seismology.Computers in Geosciences, 32, pp. 371-381.

Fernández, M. Y Rojas, W. (2001). Amenaza sísmica y tsunamis en el territorio de Costa Rica. Libro conmemorativo del 30 aniversario de la Escuela Centroamericana de Geología. Editorial Universidad de Costa Rica, Universidad de Costa Rica.

GCMT (2018). Global CMT Catalog. Recuperado de: http://www.globalcmt.org/CMTsearch.html.

Havskov and Ottemoller (1999). SeisAn Earthquake analysis software. Seis. Res. Lett., 70, 1999.

Laboratorio de Ingenieria Sismica (2018). http://www.lis.ucr.ac.cr.

Lupi, M., Fuchs, F., and Pacheco, J. F. (2014). Fault reactivation due to the M7.6 Nicoya earthquake at the Turrialba-Irazú volcanic complex, Costa Rica: Effects of dynamic stress triggering, Geophys. Res. Lett., 41, 4142-4148, doi: 10.1002/2014GL059942.

Marshall, J.S., Fisher, D.M. \& Gardner, T.W. (2000). Central Costa Rica deformed belt: kinematics of diffuse faulting across the western Panama block, Tectonics, 19, 468-492.

Meschede, M., and Barckhausen, U. (2000). Plate tectonic evolution of the Cocos-Nazca spreading center. In Silver, E.A., Kimura, G., and 
Shipley, T.H. (Eds.), Proc. ODP, Sci. Results, 170: College Station, TX (Ocean Drilling Program), 1-10 [Online]. Available from World Wide Web: <http://wwwodp.tamu.edu/publications/170_SR/VOLUME/CHAPTERSSR170_07.PDF>. Último acceso 2018-05-20.

Montero, W. (1999). El Terremoto del 4 de Marzo de 1924 ( Ms 7.0); Un gran temblor interplaca relacionado al límite incipiente entre la placa Caribe y la microplaca de Panamá, Rev. Geol. Amer. Centr., 22, 25-62.

Moya, A. (2006). Nuevo Formato de datos para el Laboratorio de Ingenieria Sismica del Instituto de Investigaciones en Ingenieria de la Universidad de Costa Rica. Ingenieria. ISSN: 1409-2441 16 (2), 63e74. San Jose, Costa Rica.

Observatorio Vulcanológico y Sismológico de Costa Rica, Universidad Nacional (2018). https://www.facebook.com/OVSICORI/. Último acceso 20 mayo, 2018.

Observatorio Vulcanológico y Sismológico de Costa Rica, Universidad Nacional (2018). http://www.ovsicori.una.ac.cr. Último acceso 19 de Mayo, 2018.

Protti, M., Güendel, F. and McNally, K. (1995). Correlation between the age of the subducting Cocos Plate and the geometry of the WadatiBenioff zone under Nicaragua and Costa Rica. En: MANN, P. (ed.): Geologic and Tectonic development of the Caribbean plate boundary in southern Central America. Geol. Soc. Amer. Spec. Paper, 295: 309-326

Quintero, R., and E. Kissling (2001). An improved P-wave velocity reference model for Costa Rica. Geofis. Int., 40, 3-19.

$\mathrm{R}$ Core Team (2013). $R$ : A language and environment for statistical computing. R Foundation for Statistical Computing, Vienna, Austria. URL http://www.R-project.org/.

Rojas, W,. Lindholm, C. \& Bungum, H. (1998). Seismic hazard analisis for the Metropolitan Area of the Central Valley, Costa Rica. Technical Report, NORSAR, Norway, 59p.

Segura, J. D., Quintero, R., Burgoa, R. B., Jiménez, U. W. (2014). Análisis de la actividad sísmica en Costa Rica durante el 2010 y resumen de los eventos sísmicos más importantes presentados en Costa Rica de 19832012. Revista Geográfica de América Central, Vol. 1, No 52, 2014. 
Snoke, J.A., Munsey, J. W., Teague, A.G., Bollinger, G.A. (1984). A program for focal mechanism determination by combined use of polarity and SV-P amplitude data. Earthquake Notes, 55, \#3, 15.

Sokos, E., Zahradník, J. (2008). ISOLA a Fortran code and a Matlab GUI to perform multiple-point source inversion of seismic data. Comput. Geosci.34, 967e977. http://dx.doi.org/10.1016/j.cageo.2007.07.005.

Sokos, E., Zahradník, J. (2013). Evaluating centroid-moment tensor uncertainty in the new version of ISOLA software. Seis. Res. Lett. 84, 656e665. http://dx.doi.org/ 10.1785/0220130002.

Zahradník, J. and Plešinger, A. (2005). Long period pulses in broadband records of near eathquakes. Bull. Seismol. Soc. Ame. 95:1928-1939. https://doi.org/10.1785/0120040210.

Wessel, P. and Smith, W.H.F. (1995). New version of the generic mapping tools released. $\operatorname{EOS~76,~} 329$. 